\title{
MFGE8 inhibits inflammasome-induced IL-1 $\beta$ production and limits postischemic cerebral injury
}

\author{
Nicolas Deroide, ${ }^{1,2}$ Xuan Li, ${ }^{1}$ Dominique Lerouet, ${ }^{3}$ Emily Van Vré, ${ }^{4}$ Lauren Baker, ${ }^{1}$ James Harrison, ${ }^{1}$ \\ Marine Poittevin, ${ }^{2}$ Leanne Masters, ${ }^{1}$ Lina Nih, ${ }^{2}$ Isabelle Margaill, ${ }^{3}$ Yoichiro Iwakura, ${ }^{5}$ \\ Bernhard Ryffel,6 Marc Pocard,2 Alain Tedgui,4 Nathalie Kubis, ${ }^{2}$ and Ziad Mallat ${ }^{1,4}$

\begin{abstract}
1Division of Cardiovascular Medicine, University of Cambridge, Addenbrooke's Hospital, Cambridge, United Kingdom. 2Institut National de la Santé et de la Recherche Médicale (Inserm) U965, Université Paris Diderot, Sorbonne Paris Cité, Assistance Publique Hôpitaux de Paris, Hôpital Lariboisière, Paris, France. 3Equipe d'Accueil EA4475, Faculté de Pharmacie, Paris, France. ${ }^{4}$ Inserm, U970 PARCC, Paris, France. ${ }^{5}$ Center for Experimental Medicine, Institute of Medical Science, University of Tokyo, Tokyo, Japan.
\end{abstract} \\ ${ }^{6}$ University of Orleans and Centre National de la Recherche Scientifique UMR7355, Orleans, France.
}

\begin{abstract}
Milk fat globule-EGF 8 (MFGE8) plays important, nonredundant roles in several biological processes, including apoptotic cell clearance, angiogenesis, and adaptive immunity. Several recent studies have reported a potential role for MFGE8 in regulation of the innate immune response; however, the precise mechanisms underlying this role are poorly understood. Here, we show that MFGE8 is an endogenous inhibitor of inflammasome-induced IL-1 $\beta$ production. MFGE8 inhibited necrotic cell-induced and ATP-dependent IL-1 $\beta$ production by macrophages through mediation of integrin $\beta_{3}$ and $\mathrm{P} 2 \mathrm{X} 7$ receptor interactions in primed cells. Itgb3 deficiency in macrophages abrogated the inhibitory effect of MFGE8 on ATP-induced IL-1 $\beta$ production. In a setting of postischemic cerebral injury in mice, MFGE8 deficiency was associated with enhanced IL-1 $\beta$ production and larger infarct size; the latter was abolished after treatment with IL-1 receptor antagonist. MFGE8 supplementation significantly dampened caspase-1 activation and IL-1 $\beta$ production and reduced infarct size in wild-type mice, but did not limit cerebral necrosis in Il1b-, Itgb3-, or P2rx7-deficient animals. In conclusion, we demonstrated that MFGE8 regulates innate immunity through inhibition of inflammasomeinduced IL-1 $\beta$ production.
\end{abstract}

\section{Introduction}

Milk fat globule-EGF 8 (MFGE8), or lactadherin, is a secretory glycoprotein containing $\mathrm{C}$ domains that bind to anionic phospholipids (1) and extracellular matrices (2), and 1 or 2 EGF-like domains with an RGD motif that binds integrins $\alpha_{v} \beta_{3}$ and $\alpha_{v} \beta_{5}$ (3). As such, MFGE8 appears to be instrumental in cell-cell interactions and has been involved in diverse physiological functions, including fertilization (4), branching morphogenesis (5), angiogenesis (6), or preservation of epithelial integrity $(7,8)$. One of the prominent functions of MFGE8 is also to link phosphatidylserine of apoptotic cells to integrins $\alpha_{v} \beta_{3}$ and $\alpha_{v} \beta_{5}$ of phagocytic cells, thereby providing a nonredundant pathway for the clearance of apoptotic cells (3, 9-11). More recent studies have shown that MFGE8 regulates adaptive immune responses in part through the promotion of tolerogenic antigen presenting cells, which facilitate the generation and activity of regulatory $\mathrm{T}$ cells, thereby restraining pathogenic T helper responses (12-14). MFGE8 also controls the intracellular processing of apoptotic self antigens into MHC-antigen complexes and limits pathogenic antigen crosspresentation (15).

The role of MFGE8 in the regulation of the innate immune response is less clear. MFGE8 expression has been associated with antiinflammatory effects in settings such as postischemic injury (16-18), experimental colitis (19), or sepsis (20). However, no particular antiinflammatory mechanism was identified, and the

Authorship note: Nicolas Deroide and Xuan Li, as well as Nathalie Kubis and Ziad Mallat, contributed equally to this work.

Conflict of interest: The authors have declared that no conflict of interest exists. Citation for this article: J Clin Invest. 2013;123(3):1176-1181. doi:10.1172/JCI65167. effects were indirectly related to the known role of MFGE8 in the clearance of apoptotic cells. Recent data suggested that MFGE8 might interfere with osteopontin binding to integrin $\alpha_{v} \beta_{3}$ and limits NF- $\mathrm{KB}$ activation in response to LPS in vitro $(19,21)$. However, it is still unknown whether this is a major pathway by which MFGE8 exerts its antiinflammatory effects, and its relevance to inflammatory settings other than sepsis is uncertain.

In the present work, we addressed the role of MFGE8 in postischemic injury. We show that MFGE8 controls postischemic cerebral injury through a previously unsuspected mechanism involving integrin $\beta_{3}$-dependent inhibition of the inflammasome.

\section{Results and Discussion}

$M F G E 8$ reduces postischemic cerebral tissue damage and inflammatory response. We first compared $\mathrm{Mfge}^{-/-}$mice and control WT littermates in a model of focal cerebral ischemia. We found that infarct size was significantly larger in $\mathrm{Mfge}^{-1 /}$ mice compared with their controls (Figure 1A), a noteworthy augmentation of $38 \%$ which was abrogated by supplementation of $\mathrm{Mfge}^{-{ }^{-/}}$with recombinant murine MFGE8 (rMFGE8) (Figure 1B). These results clearly indicate that endogenous MFGE8 is required for protection against excessive postischemic cerebral damage. We also found that supplementation of WT mice (Figure 1C) with rMFGE8 induced a significant reduction of infarct volume, in agreement with the recently reported beneficial effect of recombinant human MFGE8 in a model of cerebral injury in rats (16).

We then investigated the potential mechanisms responsible for MFGE8 protective effect. MFGE8 has been shown to alter postischemic neovascularization (6) and fibrotic tissue response 

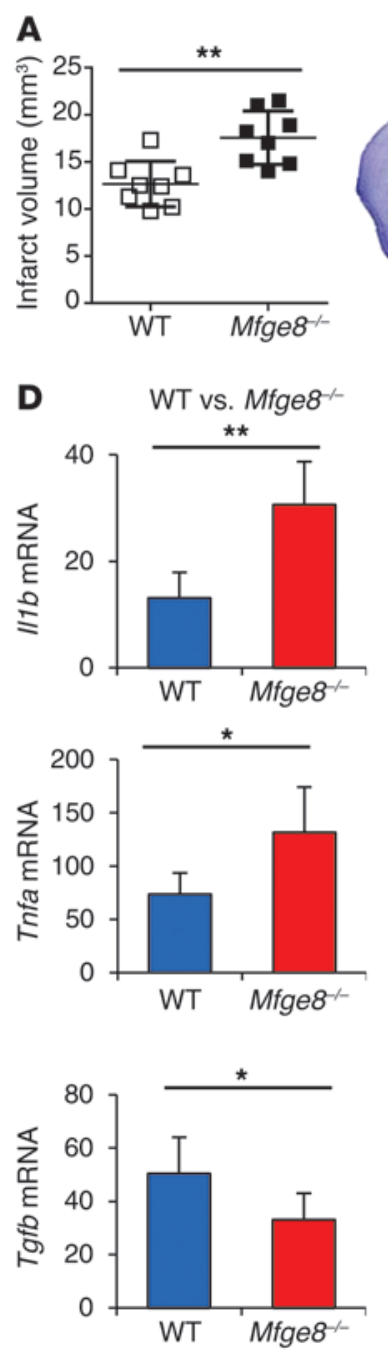

F

E $\quad$ WT + aCSF vs. rMFGE8
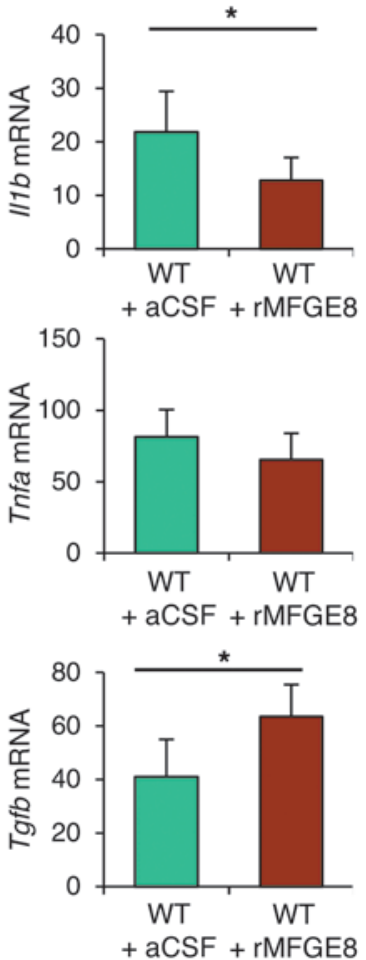

B

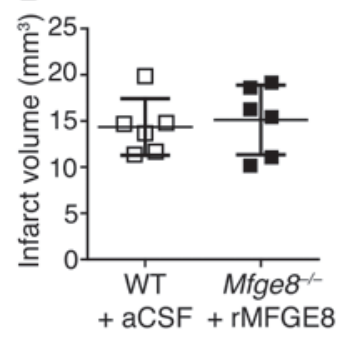

C

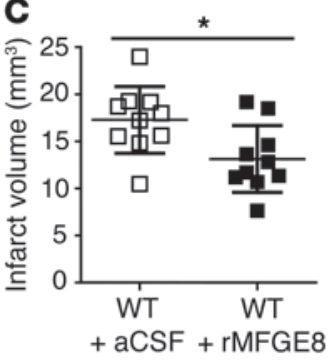

G
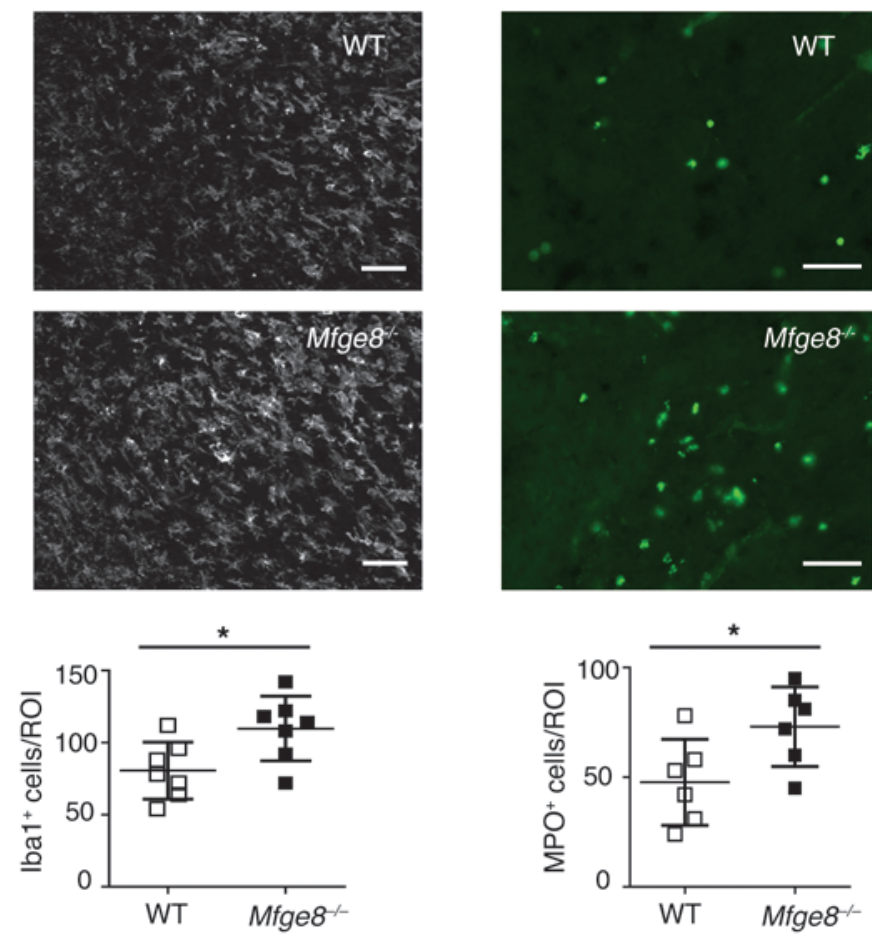

Figure 1

Effect of MFGE8 on cerebral infarct volume and brain inflammation. (A-C) Representative photomicrographs of cresyl violet staining and infarct volume quantification in WT and $\mathrm{Mfge}^{-/-}$mice, with or without supplementation with recombinant rMFGE8 (administered in artificial cerebrospinal fluid [aCSF], used as vehicle). (D and E) Cytokine expression (day 3 after artery occlusion) in brains of WT vs. Mfge $8^{-/-}$mice (D) and brains of WT mice treated with artificial cerebrospinal fluid or rMFGE8 (E). (F and G) Representative photomicrographs and quantification of microglia/ macrophages (Iba1 staining in F) and granulocyte accumulation (myeloperoxidase [MPO] staining in $\mathbf{G}$ ) in ischemic brains of WT and Mfge $^{-/-}$ mice at day 7 after artery occlusion. ${ }^{\star} P<0.05 ;{ }^{* *} P<0.01 ; n=7$ to 8 mice per group. Scale bar: $50 \mu \mathrm{m}$.

to injury (2). However, quantification of CD31-positive vascular area or collagen accumulation did not reveal any relevant difference between $M f g e 8^{-/-}$and WT mice (Supplemental Figure 1; supplemental material available online with this article; doi:10.1172/JCI65167DS1).

MFGE8 is necessary for efficient clearance of apoptotic cells. To test this hypothesis in postischemic injury, we studied the association between macrophages/microglia and apoptotic cells. We observed reduced internalization of apoptotic material in $M f g e 8^{-/-}$mice with a concomitant increase in the percentage of noningested apoptotic cells (Supplemental Figure 2A). The results are very consistent with the higher density of dead cells, including dead neurons, that accumulate in the brains of $M f g e 8^{-/-}$mice at day 7 after cerebral ischemia (Supplemental Figure 2B). Conversely, treatment with rMFGE8 reduced the accu- mulation of apoptotic cells compared with vehicle-treated mice (Supplemental Figure 2C). These results highlight the important role of MFGE8 in apoptotic cell removal during postischemic cerebral injury.

The role of apoptotic cell removal in the induction of an antiinflammatory milieu has been well explored (22). In agreement with this concept, $M \mathrm{fge}^{-/-}$mice showed a marked increase in the expression of proinflammatory mediators IL- $1 \beta$ and TNF- $\alpha$ in the ischemic brain (respectively, $+134 \%, P<0.001$, and $+78 \%, P<0.01$ ), but a decrease of antiinflammatory TGF- $\beta(-34 \%, P<0.05)$ (Figure $1 \mathrm{D})$ at day 3 after artery occlusion compared with controls. This was followed by enhanced accumulation of macrophages/ microglia (Figure 1F) and neutrophils at day 7 after ischemic injury in $\mathrm{Mfge8^{-/- }}$ mice (Figure $1 \mathrm{G}$ ). Conversely, treatment of WT mice with rMFGE8 was associated with an antiinflammatory cytokine 

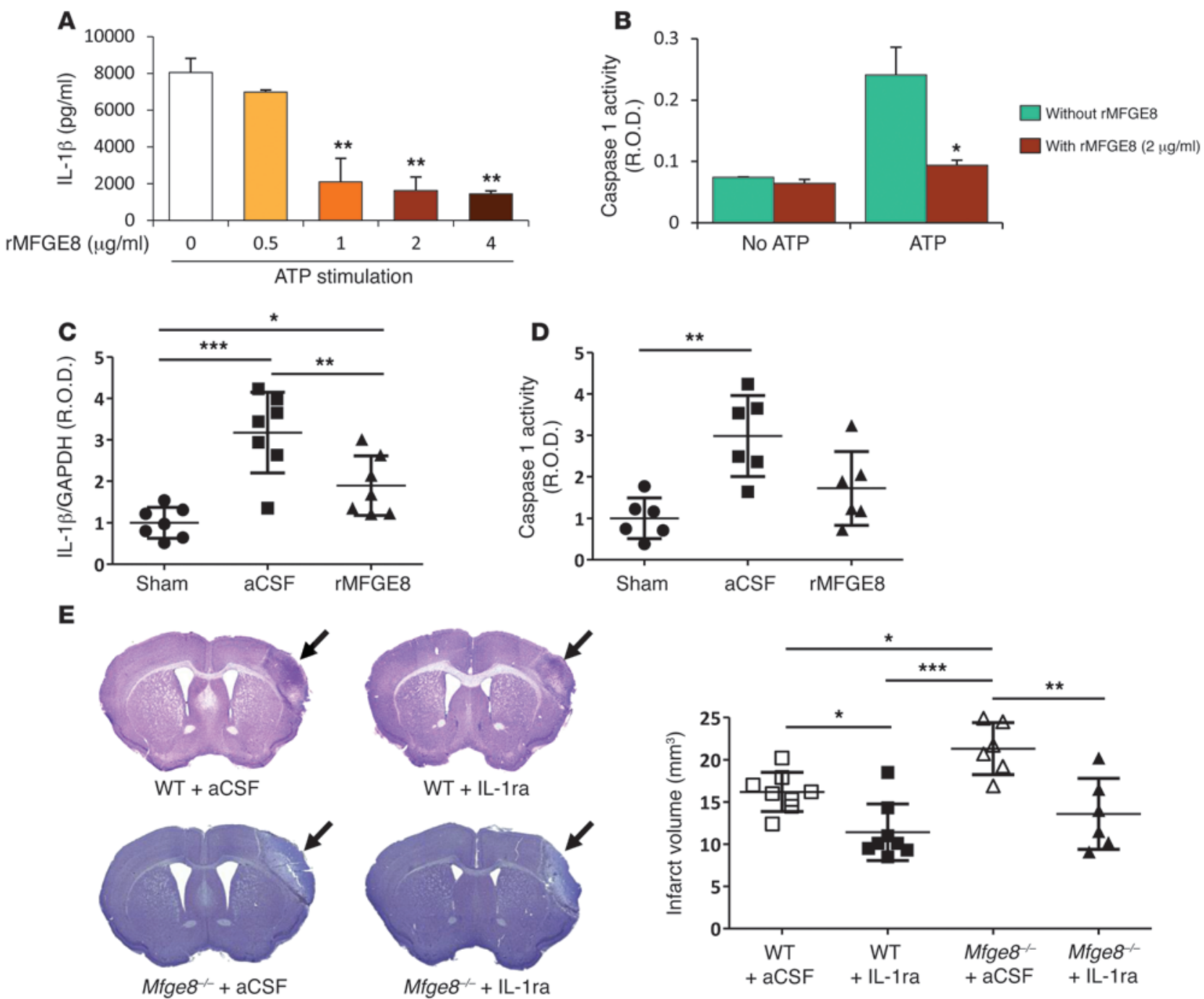

Figure 2

MFGE8 protects against postischemic cerebral injury through inhibition of inflammasome-mediated IL-1 $\beta$ production. (A and B) rMFGE8 inhibits ATP-induced IL-1 $\beta$ production (A) and caspase-1 activity (B) (see Methods). Data are representative of 3 independent experiments for in vitro experiments. Results are given as relative optic density (R.O.D.). (C and D) Cerebral ischemia significantly increased brain IL-1 $\beta$ production (C) and caspase-1 activity (D) in mice treated with artificial cerebrospinal fluid vehicle. Supplementation with rMFGE8 blunted both IL-1 $\beta$ production and the increase of caspase-1 activity. (E) Representative photomicrographs of cresyl violet staining and infarct volume quantification in WT and Mfge $8^{-/-}$ mice, with or without treatment with IL-1 receptor antagonist (IL-1 ra) (administered in artificial cerebrospinal fluid, used as vehicle). ${ }^{*} P<0.05$; ${ }^{* \star} P<0.01 ;{ }^{* \star} P<0.001 ; n=6$ to 8 mice per group for in vivo experiments.

profile, revealed by an increase of TGF- $\beta(+54 \%, P<0.05)$ and a decrease of IL-1 $\beta(-41 \%, P<0.05)$, although TNF- $\alpha$ expression was not altered (Figure 1E). Thus, the role of MFGE8 in the removal of apoptotic cells seems to be associated with the promotion of an antiinflammatory state in postischemic injury. However, the mechanistic pathways that directly mediate the protective effects of MFGE8 in this setting are still unknown.

Mfge $8^{-1-}$ antiinflammatory effect is mediated through the inflammasome/IL-1 $\beta$ pathway. Focal cerebral ischemia leads to ischemic cell death both by necrosis (especially in the core of the infarct) and apoptosis (in the penumbra). Defective removal of apoptotic cells in $M \mathrm{fge}^{-/-}$mice would also lead to secondary necrosis. Given the major effect of MFGE8 modulation on IL-1 $\beta$ expression, we hypothesized that MFGE8 might directly alter inflammasome- mediated IL-1 $\beta$ production. The rationale for this hypothesis is also based on the following observations. Necrosis leads to accumulation of extracellular ATP, a potent activator of the inflammasome through the P2X7 receptor pathway (23), and induces the production of mature IL- $1 \beta$. The inflammasome complex is activated after focal brain ischemia, and inhibition of inflammasome decreases caspase- 1 activation and IL- $1 \beta$ processing (24). The IL-1 pathway has been implicated in the pathogenesis of ischemic brain damage $(25,26)$, and our results indicate a significant increase of IL-1 $\beta$ production in ischemic brains of $\mathrm{Mfge}^{-/-}$mice compared with controls (Figure 1D and Supplemental Figure 3).

To test our hypothesis, we first used an in vitro model of BM-derived macrophages (BMDM). Incubation of LPS-primed BMDM with necrotic cells significantly induced IL- $1 \beta$ produc- 

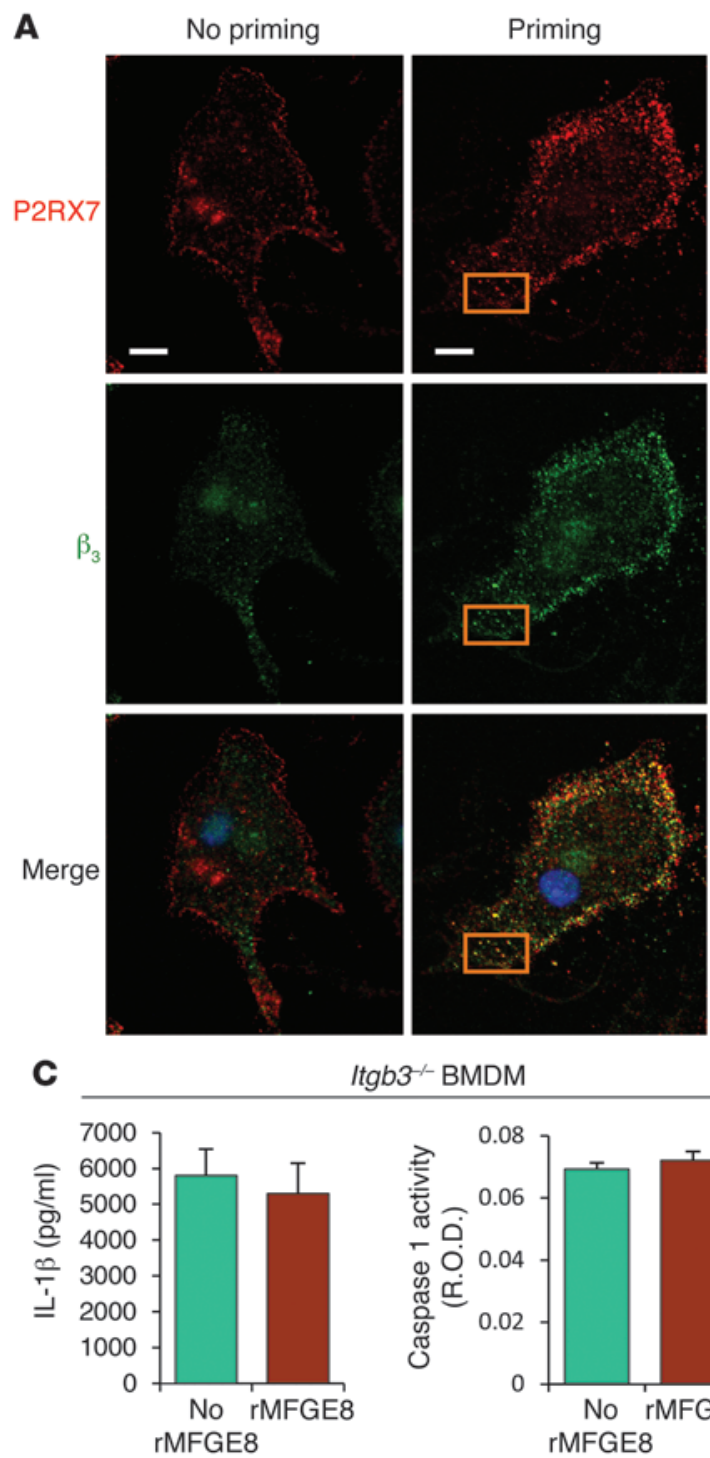

$\operatorname{ltg} 3^{-/-} \mathrm{BMDM}$

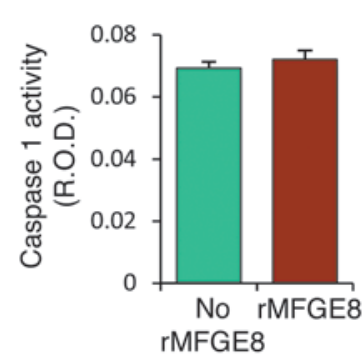

B

\section{Duolink $\beta_{3} / \mathrm{P} 2 \mathrm{RX7}$ colocalization assay}
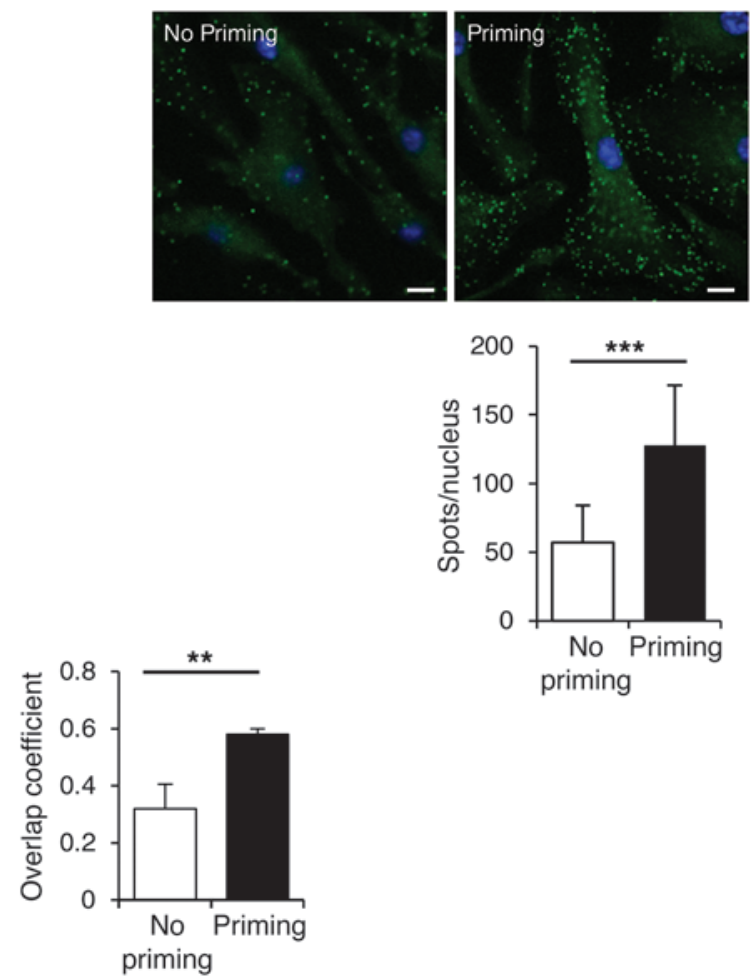

D

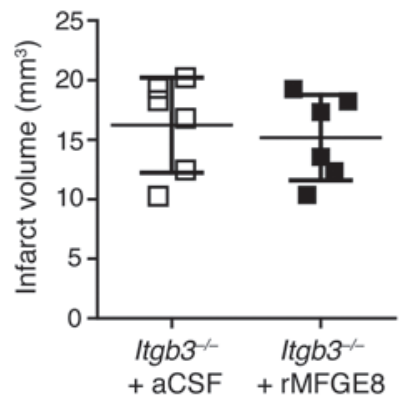

Figure 3

MFGE8 inhibits the inflammasome pathway via $\beta_{3}$ integrin. (A) Representative photomicrographs of costaining for P2RX7 and $\beta_{3}$ integrin in BMDM before and after LPS priming and analysis using confocal microscopy. A tight spatial association (quantified by the overlap coefficient) was observed between $\beta_{3}$ integrin and P2RX7 after LPS priming. (B) Duolink in situ PLA colocalization assay proving the spatial association between $\beta_{3}$ integrin and P2RX7 in BMDM after LPS priming. The spots in B indicate colocalized P2X7R and $\beta_{3}$. Data are representative of 3 independent experiments. (C) MFGE8 did not inhibit ATP-induced IL-1 $\beta$ production or caspase-1 activity in BMDM recovered from Itg $b 3^{-/-}$mice. (D and E) Quantification of infarct volume in 1 tg $b 3^{-/-}$and $P 2 r x 7^{-/-}$mice treated or not with rMFGE8; rMFGE8 did not reduce infarct volume in ltgb3 $3^{-/}$and $P 2 r \times 7^{-/-}$mice. ${ }^{* \star} P<0.01 ;{ }^{* \star} P<0.001 ; n=6$ mice per group for in vivo experiments. Scale bars: $10 \mu \mathrm{m}$.

tion, which was inhibited by incubation with apyrase, indicating an ATP-dependent process (Supplemental Figure 4). In addition, we checked using $\mathrm{Nlrp}^{3^{-/-}}$macrophages, that ATP-induced IL-1 $\beta$ production was entirely dependent on NLRP3 (Xuan Li, unpublished observations). Interestingly, we found that preincubation of LPS-primed BMDM with rMFGE8 significantly reduced IL-1 $\beta$ production in response to necrotic cells (Supplemental Figure 4) or ATP stimulation (Figure 2A). Importantly, rMFGE8 suppressed caspase- 1 activation in response to ATP (Figure 2B). Thus, MFGE8 directly alters inflammasome-mediated IL- $1 \beta$ production by macrophages in vitro. We then addressed the effect of
rMFGE8 supplementation on this process in vivo. We found that cerebral ischemia significantly increased brain IL- $1 \beta$ production (Figure 2C) and caspase-1 activity (Figure 2D). Supplementation with rMFGE8 blunted both IL-1 $\beta$ production and the increase of caspase- 1 activity after ischemia compared with vehicle-treated mice (Figure 2, C and D).

We then examined the direct contribution of the IL-1 pathway to the protective effect of MFGE8 in postischemic injury. We found that treatment with IL-1 receptor antagonist completely abrogated the increase of infarct size seen in $M f g e 8^{-/-}$mice compared with controls (Figure 2E). In addition, rMFGE8 supplementation did 
not reduce infarct size in $\mathrm{Il}-1 \mathrm{\beta}^{-/-}$mice (Supplemental Figure 5). Thus, MFGE8-dependent regulation of IL- $1 \beta$ production plays a nonredundant role in protection against postischemic injury.

$M$ fge $^{-1-}$ inhibits the inflammasome pathway via $\beta_{3}$ integrin. We then focused on the potential underlying mechanism through which MFGE8 modulates necrotic cell- or ATP-induced IL-1 $\beta$ production. $\alpha_{v} \beta_{3}$ integrin is one of the main MFGE8 receptors. P2X7 receptor is an ATP sensor and activates the NLRP3 pathway (23). As expected, ATP-induced IL- $1 \beta$ production was entirely dependent on P2X7 receptor as checked by use of P2rx $7^{-1-}$ macrophages (Xuan Li, unpublished observations). We therefore hypothesized that MFGE8 inhibits the inflammasome pathway through interaction between $\beta_{3}$ integrin and $\mathrm{P} 2 \mathrm{X} 7$ receptor. To test our hypothesis, we first studied the putative interaction between $\beta_{3}$ integrin and $\mathrm{P} 2 \mathrm{X} 7$ receptor in BMDM. Using Duolink in situ proximity ligation assay and confocal microscopy, we found a low level of colocalization between $\beta_{3}$ integrin and $\mathrm{P} 2 \mathrm{X} 7$ receptor in the absence of inflammatory stimuli. Interestingly, however, priming of BMDM with LPS increased $\beta_{3}$ expression (Supplemental Figure 6 ) and induced tight spatial association between $\beta_{3}$ integrin and $\mathrm{P} 2 \mathrm{X} 7$ receptor (Figure $3, \mathrm{~A}$ and $\mathrm{B}$ ), suggesting a potential functional role under inflammatory settings. This is supported by the finding that $\operatorname{Itg} b 3^{-/-}$BMDM produce significantly more IL-1 $\beta$ than WT cells in response to ATP stimulation (Supplemental Figure 7). We then examined the direct role of $\beta_{3}$ integrin in MFGE8-mediated IL-1 $\beta$ inhibition. We found that rMFGE8 did not alter ATPinduced IL-1 $\beta$ production or caspase- 1 activity in Itgb3 $3^{-/-}$BMDM (Figure 3C). Finally, we addressed the requirement for $\beta_{3}$ integrin and $\mathrm{P} 2 \mathrm{X} 7$ receptor pathways for MFGE8 protective effect in vivo. Interestingly, Itgb3 $3^{-/-}$and $P 2 r x 7^{-1-}$ mice were insensitive to the protective effects of rMFGE8 on infarct size (Figure 3, D and E).

MFGE8 expression is associated with immunomodulatory effects in various inflammatory settings, but the specific underlying mechanisms are still poorly described. Our work identifies a previously unsuspected antiinflammatory mechanism by which MFGE8 controls postischemic inflammatory response and injury. Necrotic cells generated after ischemic injury release extracellular ATP, which activates the inflammasome pathway and leads to IL- $1 \beta$ production. MFGE8 plays a nonredundant role in the control of this innate immune response through $\beta_{3}$ integrin and limits $\mathrm{P} 2 \mathrm{X} 7$ receptor-dependent IL- $1 \beta$ production. Our results describe a new immunomodulatory function of MFGE8 and might have broad pathophysiological and therapeutic implications, particularly in diseases associated with enhanced activation of the inflammasome.

\section{Methods}

Methods are described in detail in Supplemental Methods. All mice were fully backcrossed to a C57BL/6 background.

Permanent focal cerebral ischemia. Under anesthesia and using a small craniotomy, the middle cerebral artery (MCA) was electrocoagulated. Assessment of infarct volume was performed at day 7 after ischemia on sections stained with Cresyl violet.

In vitro stimulation, assessment of $I L-1 \beta$ production, and caspase- 1 activity. BMDM cells were primed for 6 hours with LPS unless otherwise specified. Cells were then incubated with the indicated amount of MFGE8 prior to stimulation with ATP or necrotic thymocytes. Cell media were collected for ELISA assay, caspase-1 activity (colorimetric assay), and/or Western blot.

Real-time PCR. Ready-to-use primers for IL- $1 \beta$, TNF- $\alpha$, IL-10, TGF- $\beta$, and cyclophilin A were used (QIAGEN).

Immunohistochemistry. The sources of primary antibodies are listed in Supplemental Methods. Apoptosis assay was performed using Roche In Situ Cell Death Detection Kit (Roche). Duolink in situ proximity ligation assay was performed according to the manufacturer's instructions (Olink).

Efferocytosis assessment. Association between $\mathrm{CD}^{+} 8^{+}$cells (macrophage/ microglia) and TUNEL ${ }^{+}$cells was determined on 3 magnification fields. Internalization was assessed on deconvoluted pictures using a Zeiss ApoTome (Imager Z1 with ApoTome, Carl Zeiss International).

Statistics. Statistical analyses were performed with Prism 5 software (GraphPad). All data are expressed as mean \pm SD. Comparisons of 2 different groups were analyzed by Mann-Whitney $U$ test. For more than 2 groups, we used ANOVA test with Bonferroni's post-test analysis. A $P<0.05$ was considered statistically significant.

Study approval. Experiments were performed under French Ministry of Agriculture permit no. 02934. The study was also approved by the Home Office, PPL 80/2426, United Kingdom.

\section{Acknowledgments}

This work was supported by grants from the British Heart Foundation, Fondation pour la Recherche Médicale, France, and by an ERC Starting Grant (to Z. Mallat). We are indebted to Feriel Azibani and Stéphane Potteaux for their help and advice.

Received for publication June 4, 2012, and accepted in revised form December 5, 2012.

Address correspondence to: Ziad Mallat, Department of Medicine, University of Cambridge, Addenbrooke's Hospital, West Forvie Building, Robinson Way, Cambridge CB2 0SZ, United Kingdom. Phone: 44.1223.768678; Fax: 44.1223.746962; E-mail: zm255@ medchl.cam.ac.uk.
1. Raymond A, Ensslin MA, Shur BD. SED1/ MFG-E8: a bi-motif protein that orchestrates diverse cellular interactions. J Cell Biochem. 2009; 106(6):957-966

2. Atabai K, et al. Mfge8 diminishes the severity of tissue fibrosis in mice by binding and targeting collagen for uptake by macrophages. J Clin Invest. 2009; 119(12):3713-3722.

3. Hanayama R, Tanaka M, Miwa K, Shinohara A, Iwamatsu A, Nagata S. Identification of a factor that links apoptotic cells to phagocytes. Nature. 2002; 417(6885):182-187.

4. Ensslin MA, Shur BD. Identification of mouse sperm SED1, a bimotif EGF repeat and discoidindomain protein involved in sperm-egg binding. Cell. 2003;114(4):405-417.

5. Ensslin MA, Shur BD. The EGF repeat and discoidin domain protein, SED1/MFG-E8, is required for mammary gland branching morphogenesis. Proc Natl Acad Sci U S A. 2007;104(8):2715-2720.

6. Silvestre JS, et al. Lactadherin promotes VEGFdependent neovascularization. Nat Med. 2005; 11(5):499-506.

7. Bu HF, et al. Milk fat globule-EGF factor $8 /$ lactadherin plays a crucial role in maintenance and repair of murine intestinal epithelium. J Clin Invest. 2007; 117(12):3673-3683.

8. Raymond AS, Shur BD. A novel role for SED1 (MFG-E8) in maintaining the integrity of the epididymal epithelium. J Cell Sci. 2009;122(pt 6):849-858.

9. Hanayama R, et al. Autoimmune disease and impaired uptake of apoptotic cells in MFG-E8-deficient mice. Science. 2004;304(5674):1147-1150.

10. Kranich J, et al. Engulfment of cerebral apoptotic bodies controls the course of prion disease in a mouse strain-dependent manner. J Exp Med. 2010;
207(10):2271-2281

11. Nandrot EF, Anand M, Almeida D, Atabai K, Sheppard D, Finnemann SC. Essential role for MFGE8 as ligand for alphavbeta5 integrin in diurnal retinal phagocytosis. Proc Natl Acad Sci U S A. 2007; 104(29):12005-12010.

12. Jinushi M, Nakazaki Y, Dougan M, Carrasco DR, Mihm M, Dranoff G. MFG-E8-mediated uptake of apoptotic cells by APCs links the pro- and antiinflammatory activities of GM-CSF. J Clin Invest. 2007;117(7):1902-1913.

13. Jinushi $M$, et al. Milk fat globule epidermal growth factor-8 blockade triggers tumor destruction through coordinated cell-autonomous and immune-mediated mechanisms. J Exp Med. 2009;206(6):1317-1326.

14. Ait-Oufella $\mathrm{H}$, et al. Lactadherin-defciency induces apoptotic cell accumulation, alters the regulatory 
immune response, and accelerates atherosclerosis in mice. Circulation. 2007;115(16):2168-2177.

15. Peng Y, Elkon KB. Autoimmunity in MFG-E8-deficient mice is associated with altered trafficking and enhanced cross-presentation of apoptotic cell antigens. J Clin Invest. 2011;121(6):2221-2241.

16. Cheyuo C, et al. Recombinant human MFG-E8 attenuates cerebral ischemic injury: Its role in antiinflammation and anti-apoptosis. Neuropharmacology. 2012;62(2):890-900.

17. Cui $\mathrm{T}$, et al. Milk fat globule epidermal growth factor 8 attenuates acute lung injury in mice after intestinal ischemia and reperfusion. Am J Respir Crit Care Med. 2010;181(3):238-246.

18. Matsuda A, et al. Protective effect of milk fat globule-epidermal growth factor-factor VIII after renal ischemia-reperfusion injury in mice. Crit Care Med. 2011;39(9):2039-2047.

19. Aziz MM, et al. MFG-E8 attenuates intestinal inflammation in murine experimental colitis by modulating osteopontin-dependent alphavbeta3 integrin signaling. J Immunol. 2009;182(11):7222-7232.

20. Miksa M, Wu R, Dong W, Das P, Yang D, Wang P. Dendritic cell-derived exosomes containing milk fat globule epidermal growth factor-factor VIII attenuate proinflammatory responses in sepsis. Shock. 2006;25(6):586-593.

21. Aziz M, et al. Pre-treatment of recombinant mouse MFG-E8 downregulates LPS-induced TNF-alpha production in macrophages via STAT3-mediated SOCS3 activation. PLoS One. 2011;6(11):e27685.

22. Savill J, Fadok V. Corpse clearance defines the mean- ing of cell death. Nature. 2000;407(6805):784-788

23. Schroder K, Tschopp J. The inflammasomes. Cell. 2010;140(6):821-832

24. Abulafia DP, de Rivero Vaccari JP, Lozano JD, Lotocki G, Keane RW, Dietrich WD. Inhibition of the inflammasome complex reduces the inflammatory response after thromboembolic stroke in mice. J Cereb Blood Flow Metab. 2009;29(3):534-544.

25. Boutin H, LeFeuvre RA, Horai R, Asano M, Iwakura Y, Rothwell NJ. Role of IL-1alpha and IL1 beta in ischemic brain damage. J Neurosci. 2001; 21(15):5528-5534.

26. Banwell V, Sena ES, Macleod MR. Systematic review and stratified meta-analysis of the efficacy of interleukin-1 receptor antagonist in animal models of stroke. J Stroke Cerebrovasc Dis. 2009;18(4):269-276. 